\title{
La Création du Néologisme Français dans le Dictionnaire Général Bilingue Arabe-Français Moderne
}

\author{
Ibada Hilal, Département de Langue et Littérature Françaises,
} Université de Jordanie, Amman, Jordanie (ibada01@yahoo.fr)

\begin{abstract}
Résumé: Le présent article a pour objet d'étudier pourquoi et comment le dictionnaire général bilingue arabe-français moderne pourrait provoquer des néologismes français comme correspondants à des entrées et sous-entrées arabes étiquetées d'un domaine scientifique ou technique. Pour ce faire, la présente étude se propose de travailler sur la lettre [th] du dictionnaire général bilingue arabe-français Abdelnour moderne daté de l'année 2002. Ce dictionnaire est considéré comme l'un des dictionnaires généraux bilingues arabe-français les plus utilisés dans le monde arabe.
\end{abstract}

Mots-clés: NÉOLOGISME FRANÇAIS, DICTIONNAIRE GÉNÉRAL BILINGUE ARABEFRANÇAIS MODERNE, UNITÉ LEXICALE, PALÉOLOGISME, CORPUS, ÉCART CULTUREL

\begin{abstract}
Creation of French Neologism in the Modern Arabic-French General Bilingual Dictionary. The purpose of this article is to investigate why and how the modern Arabic-French general bilingual dictionary could induce French neologisms corresponding to labeled Arabic entries and subentries of a scientific or technical domain. To do this, the present study proposes to work on the letter [th] of the Arabic-French general bilingual dictionary Abdelnour Modern (2002). This dictionary is considered as one of the most used Arabic-French general bilingual dictionaries in the Arab World.
\end{abstract}

Keywords: FRENCH NEOLOGISM, MODERN ARABIC-FRENCH GENERAL BILINGUAL DICTIONARY, LEXICAL UNIT, PALEOLOGISM, CORPUS, CULTURE GAP

\section{Introduction}

Le néologisme est le produit linguistique qui résulte du processus de la néologie. Cette dernière est, par ailleurs, «le processus de formation de nouvelles unités lexicales. Selon les frontières qu'on veut assigner à la néologie, on se contentera de rendre compte des mots nouveaux, ou l'on englobera dans l'étude toutes les nouvelles unités de signification (mots nouveaux et nouvelles combinaisons ou expressions)» (Dubois 1994: 322). Notons, que la nouveauté en question se détermine par rapport à une période précise de la vie de la communauté linguistique (Guilbert 1973: 12). 
Il est bien connu, d'ailleurs, qu'un nombre non négligeable de néologismes arabes apparaît dans le dictionnaire général bilingue ${ }^{1}$ français-arabe moderne. Ces néologismes sont des correspondants ${ }^{2}$ arabes donnés à des entrées ${ }^{3}$ ou sous-entrées françaises étant originairement des termes scientifiques et techniques $^{4}$. En effet, la terminologie scientifique et technique française, qui est en évolution constante, pénètre de plus en plus dans la langue générale et entre par la suite dans le dictionnaire général. Le correspondant arabe auxdites entrées et sous-entrées françaises n'existant pas encore en langue arabe, le lexicographe bilingue français-arabe fait, le plus souvent, appel à la création du correspondant arabe. Le dictionnaire général bilingue français-arabe Abdelnour détaillé, à titre d'exemple, va même jusqu'à consacrer un astérisque $\left(^{*}\right)$ au correspondant arabe qui constitue un néologisme créé par le lexicographe luimême ${ }^{5}$. De toute évidence, une partie importante de ces néologismes sont proposés comme correspondants arabes à des entrées ou sous-entrées françaises étiquetées d'un domaine donné.

Ainsi, si le dictionnaire général bilingue français-arabe moderne joue un rôle dans la création de néologismes arabes et pour la raison primordiale que la culture française représentée par la langue française prévaut à l'époque moderne contre la culture arabe représentée par la langue arabe, le dictionnaire général bilingue moderne qui part, cette fois-ci, de l'arabe vers le français pourrait-il, de la même manière, provoquer des néologismes français en proposant un correspondant français non existant en langue française à une entrée ou sous-entrée arabe étiquetée d'un domaine technique ou scientifique? Si oui: quelles en sont les raisons, quels sont les types du produit néologique émergé et quels sont les types de néologie repérés ainsi que les procédés de formation des néologismes en question?

Pour répondre à ces questions, nous avons choisi de travailler sur le dictionnaire général bilingue arabe-français Abdelnour moderne. Ce dictionnaire est élaboré par Jabbour Abdelnour et édité en 2002 par Dar El-Ilm Lilmalayin à Beyrouth. Ledit dictionnaire prétend confronter la langue arabe moderne à la langue française moderne. Il est, d'ailleurs, l'un des dictionnaires généraux bilingues arabe-français les plus utilisés dans le monde arabe; ce dictionnaire étant exhaustif (Abdelnour 2002: 8) et pratique à consulter.

Cela dit, comme notre travail ne vise pas l'exhaustivité, nous nous sommes décidée d'étudier les correspondants français des entrées et sous-entrées arabes étiquetées d'un domaine technique ou scientifique de la lettre $\doteq$ [th] dudit dictionnaire. Cette dernière est constituée d'un nombre de pages assez raisonnable à étudier: 16 pages. Ces 16 pages renferment, en outre, un bon nombre d'entrées et sous-entrées étiquetées d'un domaine scientifique et technique, en l'occurrence 170. Celles-ci sont réparties entre plusieurs domaines. Parmi ces domaines il y a notamment la philosophie, le commerce, le droit et la justice, les mathématiques, la chimie, la biologie, la botanique, l'entomologie, la médecine et l'astronomie.

Pour repérer les éventuels néologismes français ${ }^{6}$, nous avons cherché les correspondants français desdites 170 entrées et sous-entrées arabes dans le dic- 
tionnaire général monolingue; celui-ci demeurant le facteur de reconnaissance des néologismes par rapport à la langue générale (Sablayrolles 2000: 254-255). En effet, ces derniers ne devraient normalement pas figurer dans un dictionnaire qui représente la langue de l'usage tel que le dictionnaire général monolingue. Cependant, vu les imperfections du dictionnaire, nous avons recouru à deux dictionnaires généraux monolingues français modernes à la fois, i.e. Le Petit Robert et Le Petit Larousse.

Pour pouvoir vérifier les néologismes dans le dictionnaire général monolingue par rapport à l'année 2002; celle-ci étant l'année de l'édition du dictionnaire Abdelnour moderne en question, les éditions des deux dictionnaires français choisis sont évidemment datées de cette année même, i.e. 2002.

Nous avons ainsi retenu les néologismes non trouvés dans aucun des deux dictionnaires généraux concernés. Et contrairement à toute attente, nous avons bien trouvé des néologismes français donnés comme correspondants à des entrées et sous-entrées arabes. Nous en avons, en effet, trouvé quatre. Malgré la médiocrité du nombre des néologismes trouvés, la simple existence de néologismes français donnés comme correspondants à des entrées et sous-entrées arabes étiquetées stimulent forcément la curiosité; la culture française représentée par la langue française prévalant à l'époque moderne contre la culture arabe représentée par la langue arabe!

Les quatre néologismes relevés sont, ainsi, les suivants: fièvre octane donné comme correspondant à la sous-entrée arabe حمى ثمانية [hummā thumāniyya] de même que pennisète, sénebière et thapsie successivement donnés comme corre-

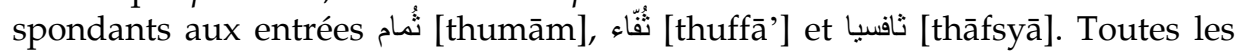
trois entrées appartiennent au domaine botanique alors que la sous-entrée relève du médical. Peut-être, dans la lettre ث [th], le taux déjà élevé d'entrées et sous-entrées étiquetées du domaine botanique par rapport à celles qui sont étiquetées de tout autre domaine scientifique et technique $(\approx 25 \%)$ a-t-il conduit à un tel résultat.

\section{Analyse}

\subsection{Types du produit néologique émergé}

Bien que le néologisme ne corresponde pas seulement à une nouvelle unité lexicale mais aussi à une nouvelle collocation (Gaudin et Guespin 2000: 228) ou combinatoire syntaxique (Mel'čuk et al. 1995: 117), tous les quatre néologismes retenus par notre étude sont des unités lexicales. Cela montre vraisemblablement qu'on a beaucoup plus besoin de créer des néologismes pour évaluer et nommer des objets - concrets et abstraits - que pour exprimer des idées. En effet, l'unité lexicale sert à dénommer des objets, des qualités, des comportements et actions et des états. Les combinatoires syntaxique et lexicale servent, par contre, à imposer certaines contraintes s'exerçant sur l'association des unités en discours. 
Trois des quatre néologismes sont, par ailleurs, des unités lexicales simples et un seul néologisme constitue une unité lexicale complexe. En effet, chacune des unités lexicales; pennisète, sénebière et thapsie, correspond à ce qu'on appelle traditionnellement le mot, c'est-à-dire un élément linguistique significatif entre deux blancs. Le quatrième néologisme; fièvre octane, est par contre composé de deux éléments lexicaux autonomes: fièvre et octane. Ceux-ci se comportent comme une unité du point de vue de la classe de mots (la catégorie grammaticale), en l'occurrence, substantive. L'unité lexicale complexe dépasse généralement le mot traditionnel pour correspondre au syntagme lexicalisé.

Tous les quatre néologismes sont, d'ailleurs, des substantifs. Cela serait dû au fait qu'on a plus besoin de dénommer des objets concrets et abstraits (concepts) - exprimés par le substantif - que des comportements et actions exprimés par le verbe - ou des qualités - exprimées par l'adjectif - ou des états - exprimés par l'adverbe (Sablayrolles 2000: 319).

\subsection{Types de néologie repérés et procédés de formation des néologismes en question}

Les quatre néologismes concernés sont créés par matrices internes ${ }^{7}$, c'est-à-dire produits dans le même système linguistique, en l'occurrence, le français. Tous les quatre néologismes seraient, en effet, empruntés à une forme ancienne de la langue française; le français du XIXe siècle. Lesdits néologismes constitueraient, ainsi, des emprunts internes, c'est-à-dire des emprunts non pas à d'autres systèmes linguistiques, à des langues étrangères. L'emprunt interne en jeu est effectué entre deux formes de la langue française; une est plus ancienne que l'autre. Ce type d'emprunts internes est, d'ailleurs, appelé le paléologisme (Sablayrolles 2000: 235).

De fait, aucun des quatre néologismes en question ne figure non plus dans le dictionnaire spécialisé daté toujours de l'année 2002. Ils ne figurent même pas dans le dictionnaire général ou spécialisé daté des XXIe ou XXe siècles. Cependant et contrairement à toute attente, nous avons trouvé lesdits néologismes dans des dictionnaires datés du XIXe siècle. Ainsi, fièvre octane, thapsie et sénebière figurent dans le fameux Littré8, qui est un dictionnaire général monolingue élaboré par Emile Littré et publié en 1889 par Hachette, alors que pennisète figure dans le Complément du dictionnaire de l'Académie Française 9 , qui est un dictionnaire spécialisé monolingue ${ }^{10}$ élaboré sous la direction de Louis Barré en 1842. Ce dernier néologisme ne figure même pas dans Le Littré.

Comme le néologisme pennisète ne figure que dans un dictionnaire spécialisé, il ne s'agit donc pas seulement de paléologisme créé par emprunt interne mais aussi de néologisme sémantique créé par changement de sens; plus précisément par extension de sens (Pruvost et Sablayrolles 2003: 112). En effet, ledit néologisme serait déterminologisé (passé de la langue spécialisée à la langue générale). Et pour qu'il soit déterminologisé, il doit forcément avoir subi une extension sémantique. 


\subsection{Raisons d'émergence du néologisme repéré}

Deux raisons principales seraient l'origine de l'apparition des quatre néologismes en jeu:

(1) L'absence de corpus lors de l'élaboration du dictionnaire dans le monde arabe

Aucun des deux entrées arabes; ثافسيا [thāfsyā] à laquelle correspond le néologisme thapsie ou حمى ثمانية [hummā thumāniyya] à laquelle correspond le néologisme fièvre octane, n'auraient existé en arabe en 2002. Elles ne figurent, en effet, dans aucun dictionnaire monolingue arabe général ni spécialisé de ladite année; le dictionnaire monolingue étant le représentant de la langue à un moment donné.

A notre grande surprise, pourtant, nous avons trouvé [thāfsyā] dans un dictionnaire monolingue scientifique ancien datée de l'année 1832, en l'occurrence, الثرح المغني [Al-sharh al-mughnī]. حمى ثمانية [hummā thumāniyya], qui ne figure même pas dans le dictionnaire ancien, figure par contre dans un dictionnaire bilingue français-arabe moderne. Il s'agit du dictionnaire Al-kamel al-kabir édité en $2001^{11}$.

Aussi, pour élaborer sa nomenclature, le dictionnaire bilingue arabe-français moderne se base-t-il, entre autres, sur des dictionnaires anciens et le dictionnaire bilingue français-arabe moderne. Il ne se base surtout pas sur un corpus, composé d'énoncés réels, qui représente la langue à un moment donné.

Par conséquent et comme les correspondants français des entrées ثافسيا [thāfsyā] et حمى ثمانية [hummā thumāniyya] n'existaient pas en français de 2002, le lexicographe bilingue arabe-français a fait appel à la néologie.

(2) L'écart culturel entre les deux langues concernées dans le dictionnaire bilingue en jeu, en l'occurrence, l'arabe et le français

En effet, comme «élaborer un dictionnaire bilingue, c'est mettre en relation deux cultures» (Pruvost 2002: 8) et que «dans le cas des deux langues, le français et l'arabe, il existe des différences culturelles importantes» (Cherifi 2009: 238); les deux civilisations arabe et française étant distinctes, ledit dictionnaire bilingue a recouru à la néologie pour donner un correspondant français à l'entrée arabe qui existait normalement en arabe en 2002 mais dont le correspondant français n'existait pas en français de l'année même.

C'est le cas des entrées arabes; ثنّْاء [thuffā'] à laquelle correspond le néologisme sénebière et [نُمام [thumām] à laquelle correspond le néologisme pennisète, existant normalement en langue arabe en 2002. Nous avons, effectivement, trouvé toutes les deux entrées dans le dictionnaire général monolingue arabe de l'année 2002. Toutes les deux figurent, à titre d'exemple, dans المنجد في اللغة [Al-munjed fī al-lugha al-'arabiyya al-mu'āṣira] de l'année 200112. Ce dernier est un dictionnaire général monolingue arabe prétendant traiter la langue arabe moderne. ثُّام [thumām] figure, de plus, dans المجم العربي الأساسي [Al- 
mu'jam al-'arabī al-'asāsī] de l'année 1999 et l'année 2003¹3. Ledit dictionnaire est un dictionnaire général arabe prétendant traiter la langue arabe essentielle.

Les correspondants français des deux entrées arabes en question n'existaient, par contre, pas en langue française en 2002. En effet, les deux genres de plantes que ثَّف [thuffā'] et [thumām] désignent n'étaient à priori pas connus dans la culture française en 2002.

Ainsi, face à cet écart linguistique dû à un écart culturel concernant une réalité culturelle spécifique, en l'occurrence botanique, le lexicographe bilingue arabe-français a fait appel à la néologie.

Rappelons tout de même, qu'en français du XIXe siècle, on trouvait des correspondants aux deux dites entrées arabes. En effet, les deux plantes désig-

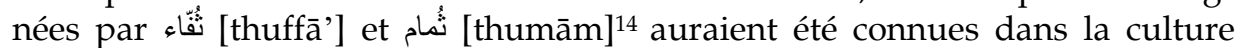
française au XIXe siècle bien qu'elles ne le soient plus en 2002.C'est peut-être la raison pour laquelle le lexicographe bilingue se serait servi du paléologisme au lieu de l'emprunt pur et simple ou de procédés d'explication.

Il est à signaler, finalement, que le lexicographe bilingue propose parfois des gloses définitionnelles à côté du néologisme français pour rendre la signification de celui-ci plus claire. Cependant, ces petites gloses ne figurent pas systématiquement à côté de chaque néologisme. De petites définitions sont, ainsi, proposées pour les deux néologismes thapsie et sénebière alors que les deux autres néologismes fièvre octane et pennisète apparaissent seuls sans le moindre commentaire.

\section{Conclusion}

Tel que le dictionnaire général bilingue français-arabe moderne, le dictionnaire général bilingue arabe-français moderne peut provoquer des néologismes comme correspondants à des entrées et sous-entrées étiquetées d'un domaine technique ou scientifique. Cependant la raison diffère. Pour le dictionnaire français-arabe, c'est le fait que la culture française prévaut à l'époque moderne contre la culture arabe à cause du développement scientifique et technique constante. Pour le dictionnaire arabe-français, c'est le manque de corpus d'une part et d'autre part l'écart culturel concernant une réalité spécifique connue par la culture arabe et non pas par la culture française; les deux civilisations arabe et française étant distinctes par nature de tout temps. La sénebière et le pennisète n'étaient, par exemple, pas connus dans la culture française en 2002 alors qu'ils l'étaient par la culture arabe. La sénebière s'utilisait en médecine douce et en cuisine alors que le pennisète dans l'élevage de volaille.

Tous les néologismes repérés sont des unités lexicales substantives comme, normalement, on a le plus besoin de créer des néologismes pour évaluer et dénommer des objets concrets et abstraits.

De plus, lesdites unités lexicales, ayant déjà fait partie d'une forme ancienne de la langue française, constituent des paléologismes créés par processus d'emprunt interne. 
Cela dit, comme nous travaillons seulement sur une seule lettre (ث[th]), notre étude ne peut faire l'objet d'une généralité. Il serait, ainsi, intéressant d'étudier d'autres lettres du même dictionnaire en question et d'étudier d'autres dictionnaires généraux bilingues arabe-français modernes.

\section{Notes}

1. Un dictionnaire général bilingue est un dictionnaire qui n'est pas spécialisé dans un domaine particulier et qui «confronte deux systèmes linguistiques et notamment deux systèmes lexicaux» (Szende 1996: 116).

2. Dans notre travail, nous utilisons le terme correspondant par opposition à équivalent; ce premier s'employant dans la langue et l'autre dans le discours.

3. Les entrées d'un dictionnaire se définissent comme des mots ou des syntagmes à définir, généralement en caractères plus gras, suivis d'un ensemble de renseignements et terminés par un blanc.

4. Qui appartiennent à des domaines de spécialité; non pas à la langue générale.

5. Or, normalement, «un mot n'entre dans le dictionnaire que parce que les lexicographes ont jugé qu'il s'était déjà bien implanté dans l'usage. Ceux-ci ne font qu'entériner cet usage, ils ne le créent pas» (Sablayrolles 2006: 143).

6. Notons que le dictionnaire bilingue en question, en l'occurrence Abdelnour moderne, ne consacre pas de signe distinctif au correspondant qui constitue un néologisme créé par le lexicographe lui-même.

7. Les termes utilisés dans le présent travail qui concernent la typologie des néologismes sont empruntés à J. Tournier et J.-F. Sablayrolles. La typologie proposée par ces derniers est fondée sur les procédés de formation des néologismes et serait la plus détaillée, cohérente et hiérarchisée des typologies.

8. Fièvre octane figure sous l'entrée octane et est défini par: «fièvre intermittente qui revient tous les huit jours». thapsie et sénebière figurent comme entrées avec les définitions respectives suivantes: «nom d'un genre d'ombelliferes à fruit oblong, échancré aux deux extrémités, muni de quatre ailes membraneuses» et "genre de plantes formé par une espèce de lepidium et une de cochlearia, cruciferes».

9. Pennisète figure comme entrée et est défini par: «genre de plantes graminées».

10. C'est un complément du Dictionnaire de l'Académie Française pour les sciences, les arts et les techniques.

11. Il est à noter que حمى ثمانية [hummā thumāniyya] figure dans le dictionnaire bilingue français-arabe comme correspondant à la sous-entrée fièvre octane. Ceci démontre, évidemment, que même le dictionnaire bilingue français-arabe ne se base pas sur un corpus représentant la langue à un moment donné. En effet, et comme nous l'avons montré plus haut dans cette étude, fièvre octane n'existait pas en français de 2001. De plus, peut-être ledit dictionnaire a-t-il fait la traduction littérale de fièvre octane pour arriver au correspondant arabe حمى ثمانية [ḥummā thumāniyya].

12. C'est l'année d'édition la plus proche de l'année concernée par la présente étude; 2002.

13. De même, ce sont les deux années d'édition les plus proches de l'année concernée par la présente étude; 2002. 
14. ثُنّْاء [thuffā'] désigne un genre de plantes crucifères. تُمَام [thumām] désigne un genre de plantes graminées.

\section{Références Bibliographiques}

\section{Dictionnaires}

Abdelnour, J. 2002. Abdelnour dictionnaire moderne arabe-français. Beyrouth: Dar El-Ilm Lilmalayin. Abdelnour, J. 2005. Abdelnour dictionnaire détaillé français-arabe. Beyrouth: Dar El-Ilm Lilmalayin. ALECSO. 1999. Al-mu' 'jam al-'arabī al-'asāsī. Paris: Larousse.

ALECSO. 2003. Al-mu' 'jam al-'arabī al-'asāsī. Paris: Larousse.

Barré, L. 1842. Complément du dictionnaire de l'Académie Française. Paris: Didot.

Dār al-mashreq. 2001. Al-munjed fĩ al-lugha al-'arabiyya al-mu 'ạsira. Beyrouth: Dār al-mashreq.

Dubois, J. 1994. Dictionnaire de linguistique et des sciences du langage. Paris: Larousse.

Kazrūtī, S. 1832. Al-sharh al-mughnī. Inde: Calcutta.

Larousse. 2002. Le Petit Larousse. Paris: Larousse.

Littré, E. 1889. Le Littré dictionnaire de la langue française. Paris: Hachette.

Reda, Y. 2001. Al-kamel al-kabir dictionnaire français-arabe. Beyrouth: Librairie du Liban.

Robert, P. 2002. Le Petit Robert. Paris: Dictionnaires Le Robert.

\section{Autres Références}

Cherifi, N. 2009. «L'écart culturel dans les dictionnaires bilingues français-arabe, arabe-français». Ela 154: 237-248.

Gaudin, F. et L. Guespin. 2000. Initiation à la lexicologie française: de la néologie aux dictionnaires. Bruxelles, Belgique: Editions Duculot.

Guilbert, L. 1973. «Théorie du néologisme». Cahiers de l'Association internationale des études françaises 25(1): 9-29.

Mel'čuk, I. et al. 1995. Introduction à la lexicologie explicative et combinatoire. Louvain-la-Neuve, Belgique: Editions Duculot.

Pruvost, J. 2002. Traduire l'écart culturel dans les dictionnaires bilingues? Traduire 2. Paris: Les belles lettres.

Pruvost, J. et J.-F. Sablayrolles. 2003. Les néologismes. Paris: Presses Universitaires de France.

Sablayrolles, J.-F. 2000. La néologie en français contemporain, examen du concept et analyse de productions néologiques récentes. Paris: Honoré Champion.

Sablayrolles, J.-F. 2006. La néologie aujourd'hui. Gruaz, C. 2006. À la recherche du mot: de la langue au discours: 141-157. Limoges: Lambert-Lucas.

Szende, Th. 1996. Problèmes d'équivalence dans les dictionnaires bilingues. Béjoint, Henri et Philippe Thoiron. 1996. Les dictionnaires bilingues: 111-126. Louvain-la-Neuve, Belgique: Editions Duculot. 